\title{
Observations of the Sun using LOFAR Baldy station
}

\author{
Dabrowski, B. P.
}

2018-10-01

Dabrowski , B P , Morosan, D E , Fallows , R A , Blaszkiewicz , L , Krankowski , A , Magdalenic , J , Vocks , C , Mann , G , Zucca , P , Sidorowicz , T , Hajduk, M , Kotulak , K, Fron , A \& Sniadkowska , K 2018 , ' Observations of the Sun using LOFAR Baldy station ' ,

Advances in Space Research , vol. 62 , no. 7 , pp. 1895-1903 . https://doi.org/10.1016/j.asr.2018.06.037

http://hdl.handle.net/10138/325203

https://doi.org/10.1016/j.asr.2018.06.037

submittedVersion

Downloaded from Helda, University of Helsinki institutional repository.

This is an electronic reprint of the original article.

This reprint may differ from the original in pagination and typographic detail.

Please cite the original version. 


\title{
Observations of the Sun using LOFAR Bałdy station
}

\author{
B.P. Dąbrowski ${ }^{\mathrm{a}, *}$, D.E. Morosan ${ }^{\mathrm{b}}$, R.A. Fallows ${ }^{\mathrm{c}}$, L. Błaszkiewicz ${ }^{\mathrm{a}, \mathrm{d}}$, \\ A. Krankowski ${ }^{\mathrm{a}}$, J. Magdalenic ${ }^{\mathrm{e}}$, C. Vocks ${ }^{\mathrm{f}}$, G. Mann ${ }^{\mathrm{f}}$, P. Zucca ${ }^{\mathrm{c}}$, \\ T. Sidorowicz ${ }^{\mathrm{a}}$, M. Hajduk ${ }^{\mathrm{a}}$, K. Kotulak ${ }^{\mathrm{a}}$, A. Frońa ${ }^{\mathrm{a}}$, K. Śniadkowska ${ }^{\mathrm{a}}$ \\ ${ }^{a}$ Space Radio-Diagnostics Research Center, University of Warmia and Mazury in Olsztyn, \\ Poland \\ ${ }^{b}$ School of Physics, Trinity College Dublin, Dublin 2, Ireland \\ ${ }^{c}$ ASTRON - The Netherlands Institute for Radio Astronomy, Dwingeloo, Netherlands \\ ${ }^{d}$ Faculty of Mathematics and Computer Sciences, University of Warmia and Mazury in \\ Olsztyn, Poland \\ ${ }^{e}$ Royal Observatory of Belgium, Brussels, Belgium \\ ${ }^{f}$ Leibniz-Institut für Astrophysik Potsdam, Potsdam, Germany
}

\begin{abstract}
We report first results of solar spectroscopic observations carried out with the Bałdy LOFAR (LOw-Frequency ARray) station, Poland from October 2016 to July 2017. During this time, we observed different types of radio emission, a type I and III radio bursts. Our observations show that the station is fully operational and it is capable to work efficiently in the single station mode for solar observations. Furthermore, in this paper we will briefly describe the observational technique and instrument capabilities and show some examples of first observations.
\end{abstract}

Keywords: telescopes, LOFAR, radio, Sun

\section{Introduction}

The LOFAR science program is very broad and it is organized in five "Key Science Projects" (KSPs). They cover early Universe research, pulsars, astroparticle physics, magnetic fields in the Universe, solar physics, space weather and sky surveys [1]. Research shows that LOFAR will also be suitable to study Jupiter-like planets and active moons [2].

\footnotetext{
* Corresponding author

Email address: bartosz.dabrowski@uwm.edu.pl (B.P. Dą̧browski)
}

Preprint submitted to Journal of ${ }^{A} T_{E} X$ Templates

April 17, 2018 
In this paper we will focus on the first solar observations from the LOFAR station in Bałdy (Poland).

\section{Radio bursts}

A large number of radio bursts were observed between October 2016 and July 2017 using LOFAR station in Bałdy, including type I and III radio bursts. These radio bursts are described in the following subsections.

\subsection{Type I bursts}

Type I radio noise storms consist of countless numerous narrowband bursts (with duration of about a second or less) over a broadband, slowly varying continuum ranging from 60 to $400 \mathrm{MHz}$. The storms can last from hours to days and they accompany complex active regions. The radiation of type I bursts is not associated with solar flares but appears to be related with the active regions with complex configuration of its photospheric magnetic field. The research shows that types I are generated by electrons accelerated to a few thermal energies by an ongoing local energy release in closed coronal magnetic structures [3, 4, 5].

\subsection{Type III radio bursts}

Type III radio bursts are the most frequently occurring radio bursts in the corona and they were first identified and classified by [6]. Type III bursts are observed in the frequency range $10 \mathrm{kHz}-1 \mathrm{GHz}$, that represents the area between lower solar corona (at the higher frequencies) up to $1 \mathrm{AU}$ (at $\mathrm{kHz}$ frequencies). Type III radio bursts can occur in groups of several bursts, with total duration of about few minutes or storms that can last for a day $[\underline{3}$. Type III bursts are signatures of electron beams accelerated in the corona traveling along the open or quasi open magnetic field lines. The majority of type III radio bursts at low frequencies have a negative drift rate which indicates that they are traveling away from the Sun. Their drift rate is given by $-0.01 f^{-1.84}$ (in $\mathrm{MHz} / \mathrm{s}$ ) [7, where $f$ is a frequency of observations in MHz. The negative sign in the formula means that we observe a drift from high to low frequency. At 
$100 \mathrm{MHz}$, the middle of LOFAR frequency band, the drift rate is $\sim 48 \mathrm{MHz} / \mathrm{s}$. [3] showed that the type III radio bursts are generated by electron beams moving with velocities of 10 to $60 \%$ of the light speed. These electron beams move up in the solar corona along magnetic field lines stimulating the plasma oscillations at the local plasma frequency [5]. The accelerated electron beams can therefore generate type III bursts via the plasma emission mechanism. The electron acceleration mechanism is most likely inducted by the magnetic reconnection 8 .

\section{The LOFAR telescope}

LOFAR, the LOw-Frequency ARray (www.lofar.org) is a radio interferometer comprising of 51 stations distributed throughout Europe. The LOFAR "core" consists of 24 stations tightly packed within $2 \mathrm{~km}$ near Exloo, Netherlands. The innermost 6 core stations are on an "artificial island" with a diameter of about $350 \mathrm{~m}$ called the Superterp. An additional 14 "remote" stations are located outside the core at a distance of up to $90 \mathrm{~km}$ from the core. "International" stations are located in Germany (6 stations), Poland (3 stations), and one station each in France, Ireland, Sweden, and UK. The baseline of the interferometer extends from about $100 \mathrm{~m}$ up to $2000 \mathrm{~km}$. This design provides unprecedenced sub-arcsecond imaging capabilities at low frequencies. All the LOFAR stations are connected by a broadband network with a data centre in Groningen, Netherlands, where the correlator is located.

A single LOFAR station comprises of a phased array system and can operate individually. It contains two fields of dipole antennas: LBA (Low Band Antennas) and HBA (High Band Antennas) operating in the frequency range of $10-90 \mathrm{MHz}$ and $110-240 \mathrm{MHz}$, respectively. Each type of station (from core to international) has a different layout and number of LBA and HBA antennas. Spatial resolution of one station is much worse than of the interferometer, beam size of the order of 2 degree in HBA [1].

The Bałdy station will focus on pulsars and solar observations when used 
in local mode [9, 10. This type of observations does not require high spatial resolution and high sensitivity. The main advantage for these observations is the wide frequency range and high time resolution of LOFAR.

\section{Receiver system for Bałdy LOFAR station}

The LOFAR field consists of 96 LBAs and 96 HBAs (Fig. 1). Each LBA antenna is composed of pair of perpendicular dipoles and each component of the HBA antennas is a system of 16 pairs of dipoles forming one element called tile. The signals of all dipoles in the tile is summed up to form output. The signal from both types of antennas is sent via coaxial cables ( $\mathrm{X}$ and $\mathrm{Y}$ polarization) with the proper delay to back-ends located in a special container. Combined and digitized information is ready to be used after being processed in the container. Depending on the type of observation, data is sent to the correlator (international mode) or to locally used computers in single station observations.

The array of antennas is fixed in position and there is no mechanical beam steering [11]. LOFAR LBAs in particular are able to see the entire sky, however their sensitivity decreases significantly below 30 degrees elevation. Using the antenna system as a phase arrayed telescope allows to digitally select the direction of the observation.

For solar research we use a special observational method called "mode 357", that allows observations in the entire frequency range of LOFAR telescope using both LBA and HBA simultaneously (more details in 12]). Mode 357 divides the frequency band into three parts:

- low band $10-90 \mathrm{MHz}$ we selected 200 subbands (54-452 with spacing of 2) centered at 10.55 to $88.28 \mathrm{MHz}$ with spacing of $0.39 \mathrm{MHz}$ and time resolution equal 1 second,

- high band $110-190 \mathrm{MHz}$ we selected 200 subbands (54-452 with spacing of 2) centered at 110.55 to $188.28 \mathrm{MHz}$ with spacing of $0.39 \mathrm{MHz}$ and time resolution equal 1 second, 
- highest band $210-250 \mathrm{MHz}$ we selected 88 subbands (54-228 with spacing of 2) centered at 210.55 to $244.53 \mathrm{MHz}$ with spacing of $0.39 \mathrm{MHz}$ and time resolution equal 1 second.

A small amount of data (beamlet statistics) is recorded at the Local Computing Unit located at the station in Bałdy but the main data string is recorded at the data center in the University of Warmia and Mazury in Olsztyn.

\section{Solar observations by LOFAR}

Solar radio observations are usually carried out at a broad range of wavelengths from microwaves $(f>3 \mathrm{GHz})$, up to dekameter waves $(f<30 \mathrm{MHz})$ [5]. There are five main types of solar radio bursts, from type I up to type V [6, 13, 14, 15, 16. In the frequency range covered by LOFAR, we can observe all these bursts. Additionally, the LOFAR frequency range $(10-240 \mathrm{MHz})$ approximately corresponds to a radial distance between 1 and 3 solar radii in the corona [17.

The LOFAR telescope can provide images of the Sun in interferometric mode [18] and dynamic spectra of the Sun in beam formed mode. Solar images require the use of core and remote stations to achieve spatial resolution of $\sim 1$ arcmin. Dynamic spectra can be obtained by a single station or a combination of LOFAR core stations. Observations of radio bursts require images over a broad frequency range in order to study location of the sources of the radio emission and their propagation. It is necessary to coordinate LOFAR solar observation campaigns with other ground and space based instruments for multi-wavelength studies [10.

The solar and space weather KSP (Key Science Project) covers studies of solar activity and the Sun's influence on interplanetary space. For this purpose, images, dynamic spectra and measurements of interplanetary scintillation are taken [19]. The first interferometric solar observations with LOFAR telescope which were a part of commissioning phase of instrument were performed on 17 March 2011, in frequency range 115-162.5 MHz. During this observation, 
a weak radio burst was registered [19]. 20] presented the first tied-array beam solar observations with the LOFAR in which images were shown simultaneously with their corresponding dynamic spectra. Type III bursts were observed during the commissioning phase on 28 February 2013. Using one of LOFAR beam formed modes (tied-array beams), high-time and frequency resolution (83 ms, $12.5 \mathrm{kHz}$ ) dynamic spectra have been recorded at 126 spatial locations sampling the Sun (for more details see [20]).

\section{Solar observations with Bałdy PL612 station}

Bałdy LOFAR station is used for interferometric observations for the International LOFAR Telescope (ILT) mode and also for local mode observations. One of the main objectives for the station in local mode are spectroscopic solar observations to obtain dynamic spectra of Sun.

From October 2016 up to January 2017, we have been performing test solar observations. The goal was to understand how the station works, how it can be controlled, how to receive dynamic spectra of the Sun etc. In February 2017, we started the regular solar monitoring in local mode. These observations were performed every Friday and Sunday, when the station became available for local projects. On Saturdays, other observations are also carried out, but if there is a high probability of occurrence of solar flares, then solar observations are carried out during all three days.

Between October 2016 and July 2017 we have observed five radio events that included type I and III radio bursts. The low number of observed radio bursts is due to the observation time in local mode being limited to two days per week and the Sun entering solar minimum (forecasts indicate that a minimum of solar activity will be at 2020). Therefore, the amount of observed radio events is currently very low. The temporal and frequency resolutions of all observations presented in this paper are $1 \mathrm{~s}$ and $0.39 \mathrm{MHz}$, respectively.

The observations consist of time-frequency-intensity data which can be presented as dynamic spectra. Signal processing from individual dipoles consist in 
that the raw data stream, sampled with $5 \mathrm{~ns} / 200 \mathrm{MHz}$ clock is passing throught a Polyphase Filter Bank, where the process of beam-forming take place. For 8 -bit mode the system is ready to create up to 488 beamlets. Each one is covering $0.19553 \mathrm{MHz}$. During the creation of dynamic spectra each channel is normalized to median value independently [1].

Radio frequency interference (RFI) was also present in our observations and these interference channels were removed. After these processing steps, we observe very clearly radio bursts in dynamic spectra originating in the solar corona.

\section{Description of the observed events}

In this section we present first results of solar spectroscopic observations carried out between October 2016 and July 2017 with Bałdy LOFAR station. At that time period we registered type I and III radio bursts. This events occurred on 22 January 2017, 14, 16, and 21 July 2017.

\subsection{Type I noise storm recorded on 22 January 2017}

The first observations of type I noise storm ware on 22 January 2017 between 10:02:16-12:02:15 UT in the frequency band 110.55-173.05 MHz (Fig. 2). The event lasted for approximately 120 minutes. The RFI and possibly reflections from the container are present in the dynamic spectrum as the Sun was very low over the horizon at this day (around 13 degrees above the horizon at noon). The RFI and reflections simultaneous with the noise storm can be seen as four horizontal red areas that occur between 10:41 UT and 10:59 UT. On the bottom panel of Fig. 2, radio flux at $139.84 \mathrm{MHz}$, extracted from the dynamic spectrum (upper panel) is presented. Figure 3 shows that the duration of structures marked "a", "b" and "c", is between 7 up to 14 seconds. Estimating the temporal width of the event is difficult, due to low temporal resolution (1 second).

In Fig. 4 we present 200-seconds long fragment from 22 January 2017 event. We can see in more detail the fine structure of the type I storm. The duration 
of chains shown on the Fig. 4 is between 9 up to $29 \mathrm{~s}$. This is a typical value for type I radio bursts [21].

During this noise storm no other significant events at other wavelengths were reported. Four active regions were observed on the Sun (NOAA 12625, 12626, 12627 and 12628) on 22 January 2017 and it is very probable that the noise storm accompanied one of these active region. Noise storms generally occur some days after the first appearance of active regions in the solar photosphere and when the regions become large and complex and are not necessarily correlated with solar flares or other events [3].

\subsection{Type I radio bursts recorded on 14 July 2017}

The type I radio bursts were recorded on 14 July 2017 in the high frequency band from $110.55 \mathrm{MHz}$ up to $243.75 \mathrm{MHz}$ (Fig. 5). We are not able to determine the beginning of the event because it had started before the beginning of our observations at 10:03:28 UT. The radio emission ended around 13:31 UT. In Fig. 6 we present 300-seconds long fragment from the event. We can see in more detail the fine structure of this event. The duration of chains shown on the Fig. 6 is between 5 up to $37 \mathrm{~s}$. This is a typical value for type I radio bursts [21. The chains have similar characteristics as the ones from 22 January 2017.

Similarly to the radio emission on 22 January 2017, during this radio event there were no significant events at other wavelengths observed. Two active regions were observed that day on the Sun (NOAA 12665 and 12666) and we believe that the radio burst were associated with one of these active region. In the low band $(10.55-88.28 \mathrm{MHz})$ we also have noticed the presence of ionospheric scintillations in the same spectra (for more informations see e.g. [11]).

\subsection{Type III radio bursts observed on 16 July 2017}

The groups of the type III radio bursts were recorded on 16 July 2017 in the high frequency band from $110.55 \mathrm{MHz}$ up to $243.75 \mathrm{MHz}$ (Figs. 7 and 8). We are not able to determine the beginning of this radio event because it started before the beginning of our observations at 09:03:30 UT. The radio event ended 
around 12:43 UT. During this event, the GOES satellite registered two C-class flares. The first one is GOES C1.3 flare which originated from the NOAA 12665 active region. Flare started at 10:32 UT, reached the maximum at 10:35 UT and ended at 10:37 UT. The second flare, GOES C1.5 originated from the same active region NOAA 12665. It started at 10:53 UT, reached the maximum at 10:57 UT and ended at 10:59 UT (Fig. 9).

\subsection{Type I and III radio bursts recorded on 21 July 2017}

The type I and III radio bursts were recorded during radio event on 21 July 2017 in the high frequency band from $110.55 \mathrm{MHz}$ up to $188.28 \mathrm{MHz}$ (Fig. 10).

Number of type III bursts was observed between around 10:10 UT and 10:19 UT, in frequency band from $110.55 \mathrm{MHz}$ up to $188.28 \mathrm{MHz}$ (Fig. 11). These type IIIs are accompanied by a type I noise storm which is observed from beginning of observations up to around 13:27 UT. No significant events at other wavelengths were observed to compare with other solar activity.

\section{Conclusions}

The first solar observations by the LOFAR station in Bałdy were carried out successfully on number of days during the year 2017. For the data analysis we used software written by Derek McKay-Bukowski and Richard Fallows for the KAIRA instrument [12. Unfortunately, up to this day, due to low solar activity, a small number of observation days and short periods of observation, we have managed to record five radio events. However, the first observations in Bałdy (presented in this paper) indicate that the LOFAR telescope is well suited for solar research at low frequencies. We are convinced that the single station observations will give a lot of interesting results soon.

\section{Acknowledgements}

The Polish LOFAR stations have been funded and supported by the Polish Ministry of Science and Higher Education. The authors thank Derek McKayBukowski (Sodankylä Geophysical Observatory and STFC Rutherford Appleton 


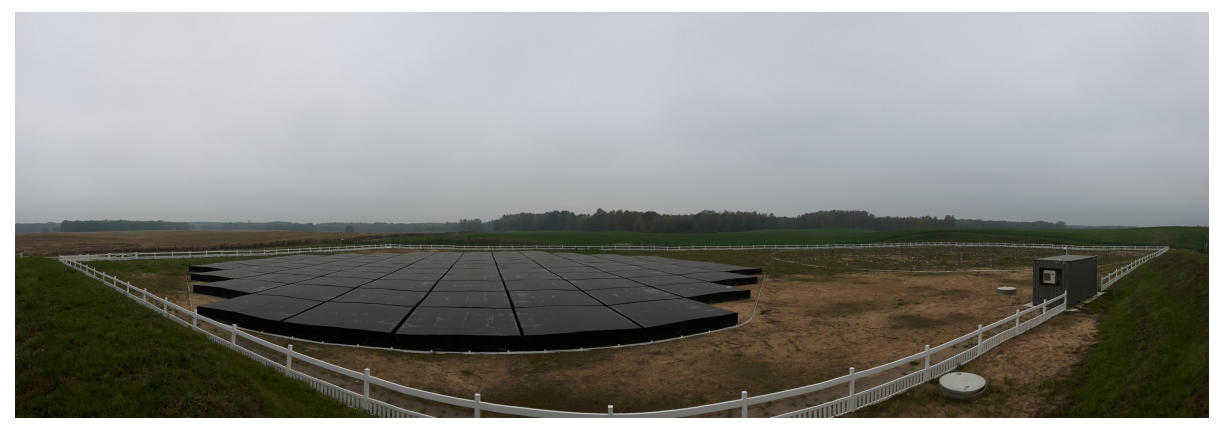

Figure 1: The LOFAR station in Bałdy. Low Band Antennas field (right), High Band Antennas field (left). Near the fence is a container (gray box) with electronics.

Laboratory, Didcot, UK) and Gottfried Mann (Leibniz-Institut für Astrophysik Potsdam) with KSP team for support in solar LOFAR research.

\section{References}

[1] M. P. van Haarlem, M. W. Wise, A. W. Gunst, G. Heald, J. P. McKean, J. W. T. Hessels, A. G. de Bruyn, R. Nijboer, J. Swinbank, R. Fallows, M. Brentjens, A. Nelles, R. Beck, H. Falcke, R. Fender, J. Hörandel, L. V. E. Koopmans, G. Mann, G. Miley, H. Röttgering, B. W. Stappers, R. A. M. J. Wijers, S. Zaroubi, M. van den Akker, A. Alexov, J. Anderson, K. Anderson, A. van Ardenne, M. Arts, A. Asgekar, I. M. Avruch, F. Batejat, L. Bähren, M. E. Bell, M. R. Bell, I. van Bemmel, P. Bennema, M. J. Bentum, G. Bernardi, P. Best, L. Bîrzan, A. Bonafede, A.-J. Boonstra, R. Braun, J. Bregman, F. Breitling, R. H. van de Brink, J. Broderick, P. C. Broekema, W. N. Brouw, M. Brüggen, H. R. Butcher, W. van Cappellen, B. Ciardi, T. Coenen, J. Conway, A. Coolen, A. Corstanje, S. Damstra, O. Davies, A. T. Deller, R.-J. Dettmar, G. van Diepen, K. Dijkstra, P. Donker, A. Doorduin, J. Dromer, M. Drost, A. van Duin, J. Eislöffel, J. van Enst, C. Ferrari, W. Frieswijk, H. Gankema, M. A. Garrett, F. de Gasperin, M. Gerbers, E. de Geus, J.-M. Grießmeier, T. Grit, P. Gruppen, J. P. Hamaker, T. Hassall, M. Hoeft, H. A. Holties, A. Horneffer, A. van der 


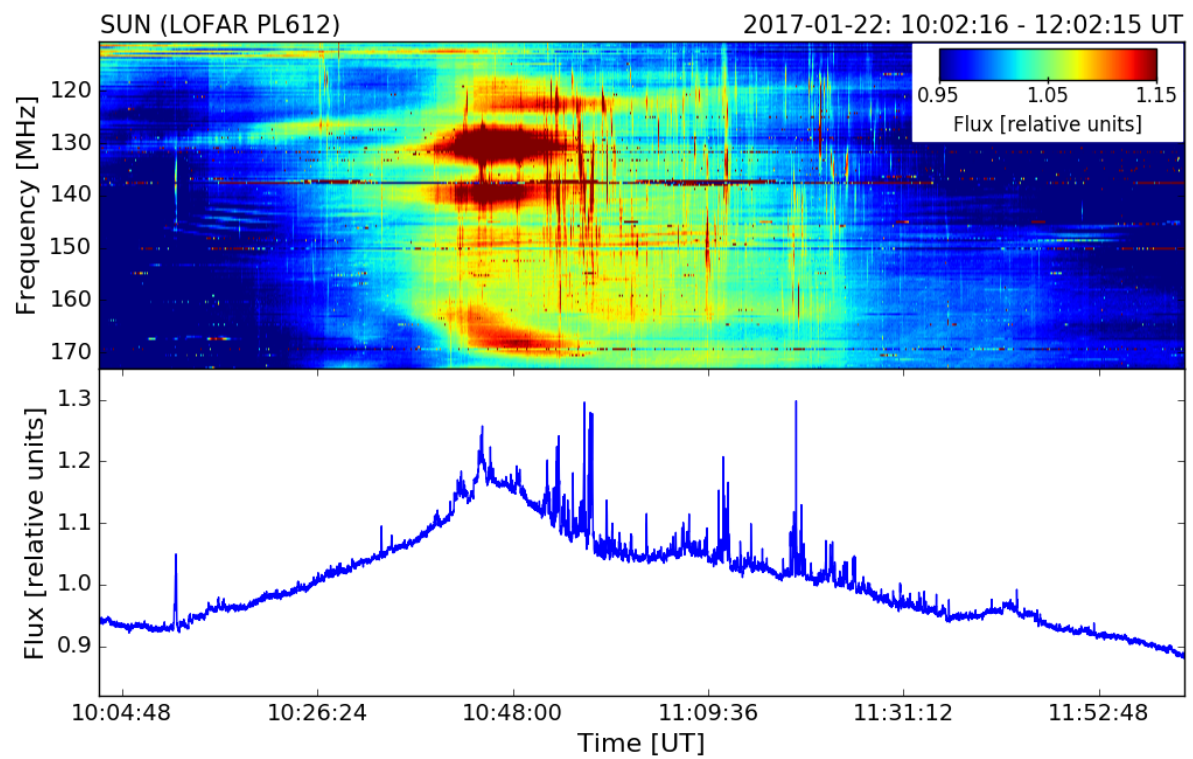

Figure 2: Upper panel: solar dynamic spectrum of the type I noise storm recorded on 22 January 2017 between 10:02:16-12:02:15 UT in frequency band 110.55-173.05 MHz with LOFAR Bałdy station. Some reflections from the container (the Sun was very low over the horizon at this day, around 13 degrees above the horizon at noon) are overlapping the noise storm, which is visible on dynamic spectrum in the form of four red areas that occur between 10:41 UT and 10:59 UT. Bottom panel: radio flux at frequency of $139.84 \mathrm{MHz}$ extracted from dynamic spectrum on the upper panel. 


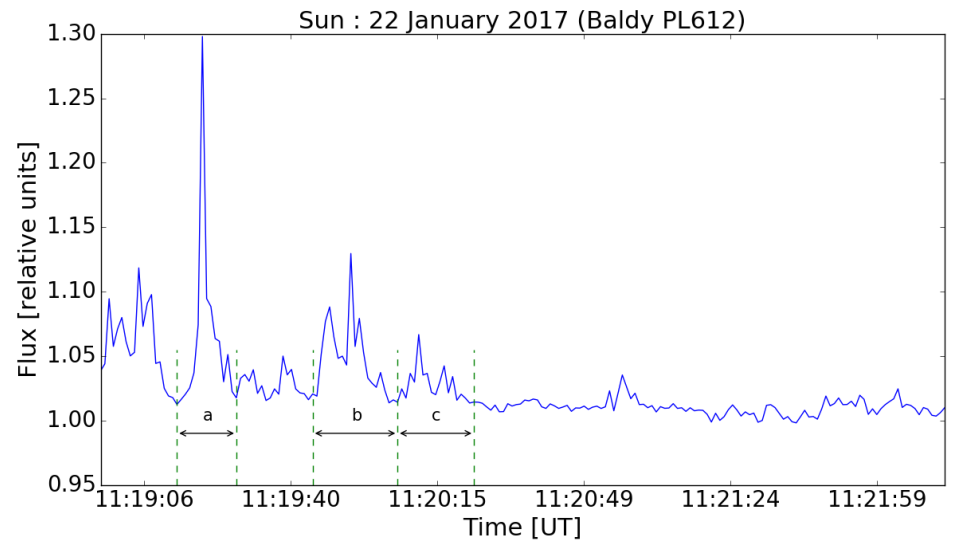

Figure 3: Radio flux recorded during the investigated radio event from 22 January 2017 between 11:18:56-11:22:16 UT in channel 139.84 MHz extracted from Fig. 2 We marked as "a", "b" and "c" the time periods where three local maxima occurred.

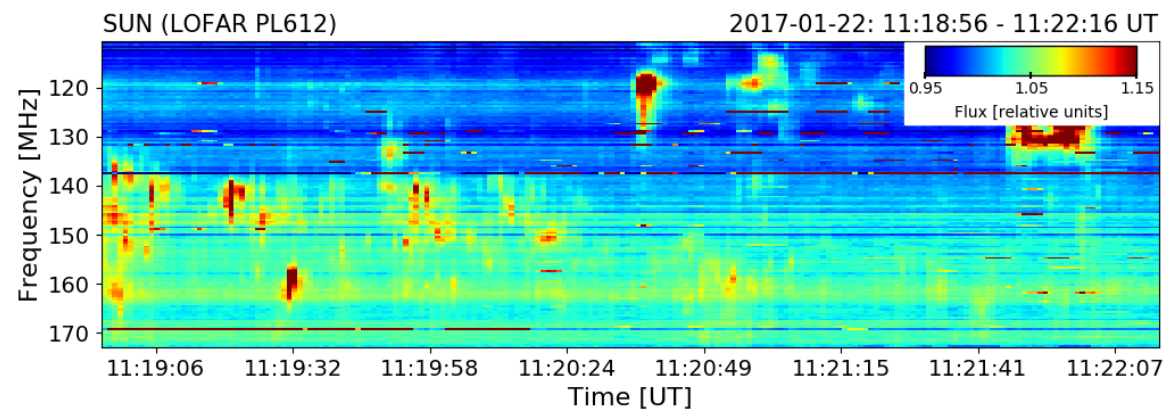

Figure 4: Solar dynamic spectrum of the type I noise storm recorded on 22 January 2017 between 11:18:56-11:22:16 UT in frequency band 110.55-173.05 MHz. 


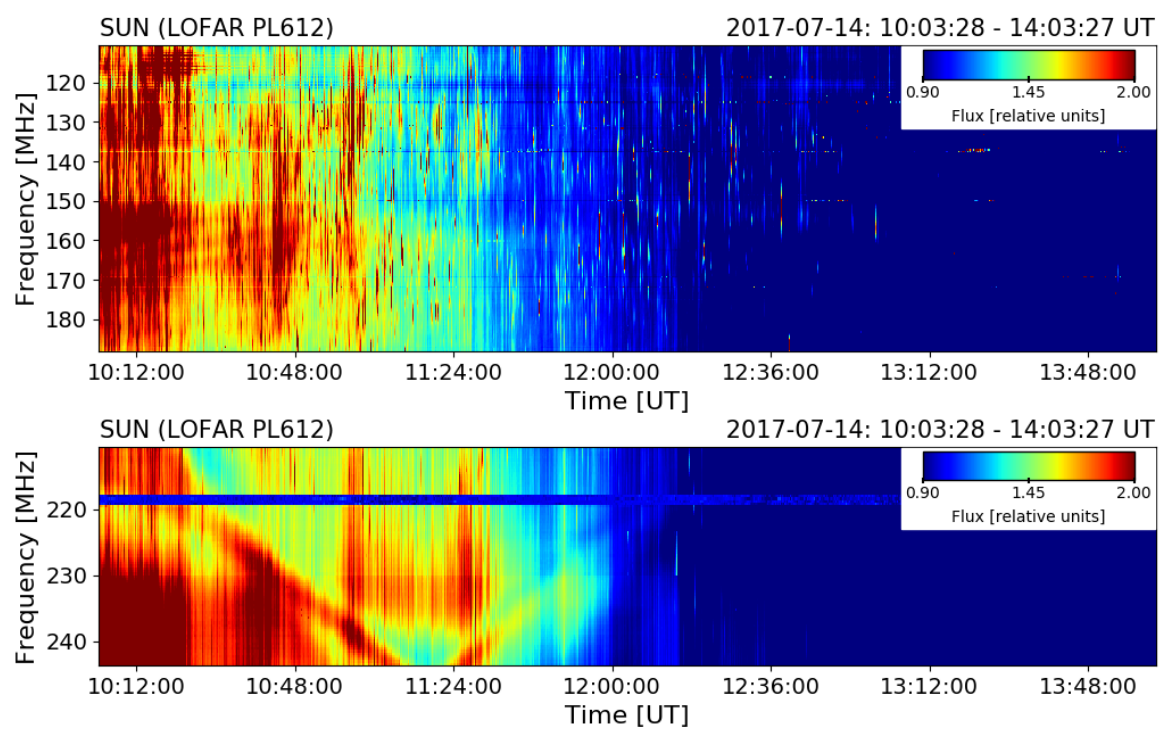

Figure 5: Solar dynamic spectrum of the type I radio burst recorded on 14 July 2017 between 10:03:28 - 14:03:27 UT in the following frequency bands: $110.55-188.28 \mathrm{MHz}$ and $210.55-$ 243.75 MHz, both HBA antennas.

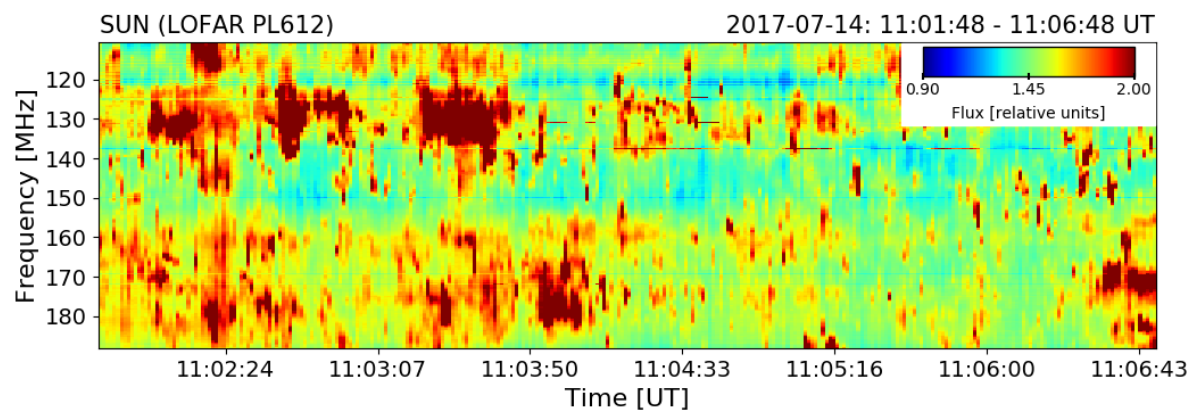

Figure 6: Solar dynamic spectrum of the type I radio burst recorded on 14 July 2017 between 11:01:48-11:06:48 UT in frequency band 110.55-188.28 MHz. 

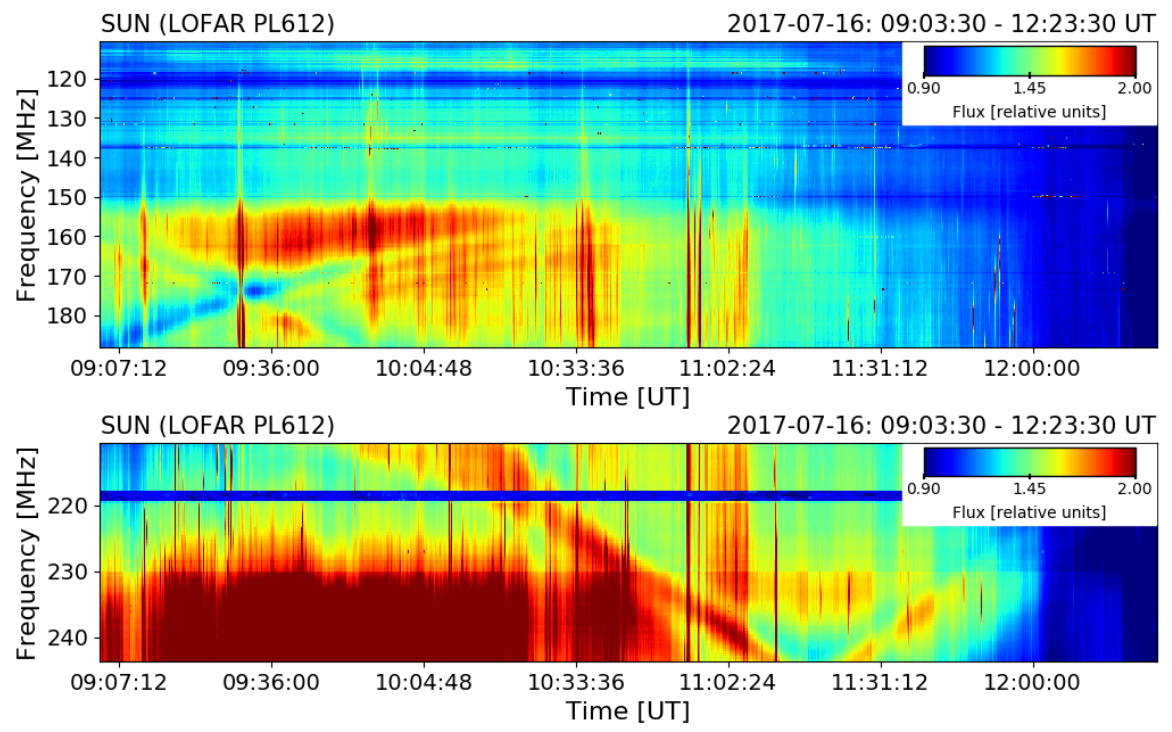

Figure 7: Solar dynamic spectrum of the type III radio burst recorded on 16 July 2017 between 09:03:30-12:23:30 UT in the following frequency bands: $110.55-188.28 \mathrm{MHz}$ and 210.55-243.75 MHz, both HBA antennas.
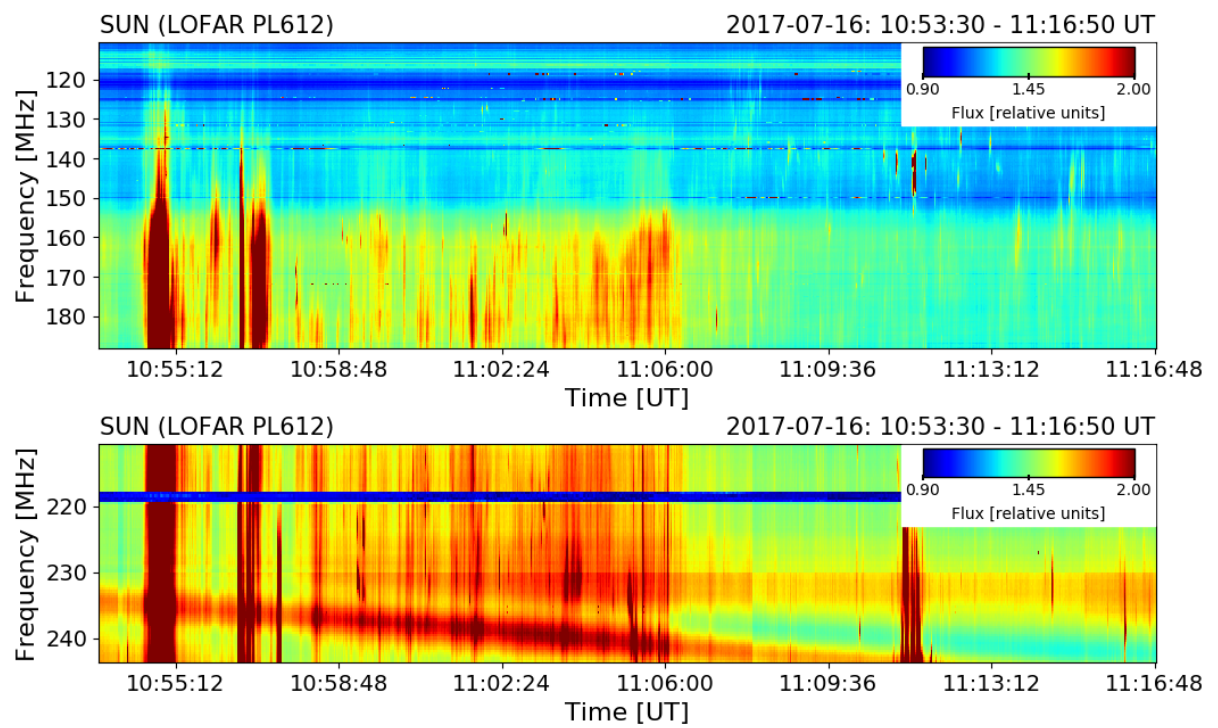

Figure 8: Solar dynamic spectrum of the type III radio event recorded on 16 July 2017 between 10:53:30-11:16:50 UT in the following frequency bands: $110.55-188.28 \mathrm{MHz}$ and $210.55-243.75 \mathrm{MHz}$, both HBA antennas. 


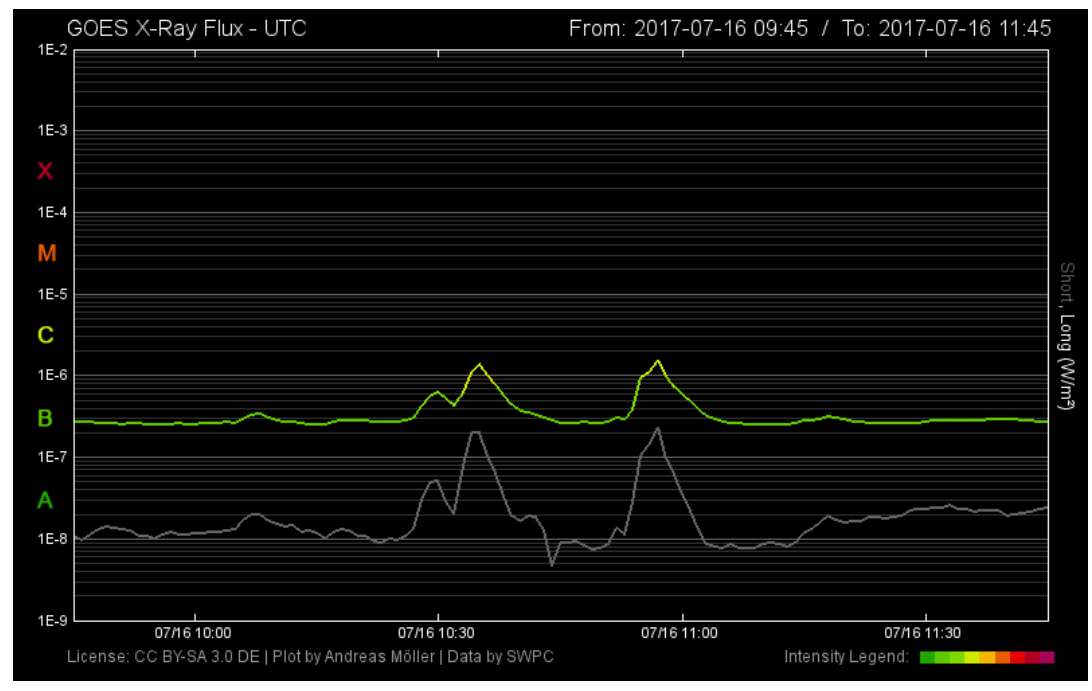

Figure 9: GOES X-ray flux recorded on 16 July 2017 between 9:46-11:45 UT. We see here two C-class flares. The first one is C1.3 and the second is C1.5 flares (see description in the text). Source: http://www.polarlicht-vorhersage.de/goes_archive.

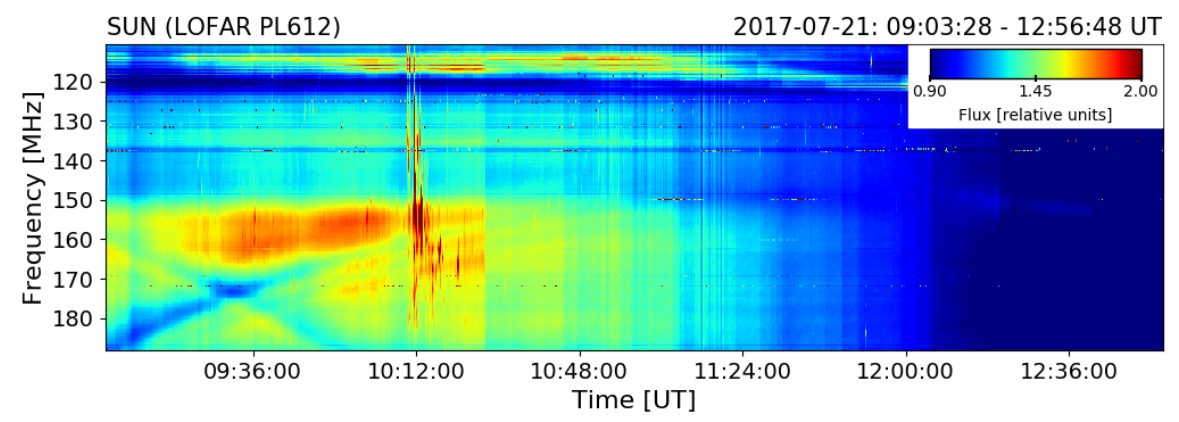

Figure 10: Solar dynamic spectrum of the type I and III radio bursts recorded on 21 July 2017 between 09:03:28-12:56:48 UT in frequency band 110.55-188.28 MHz. 


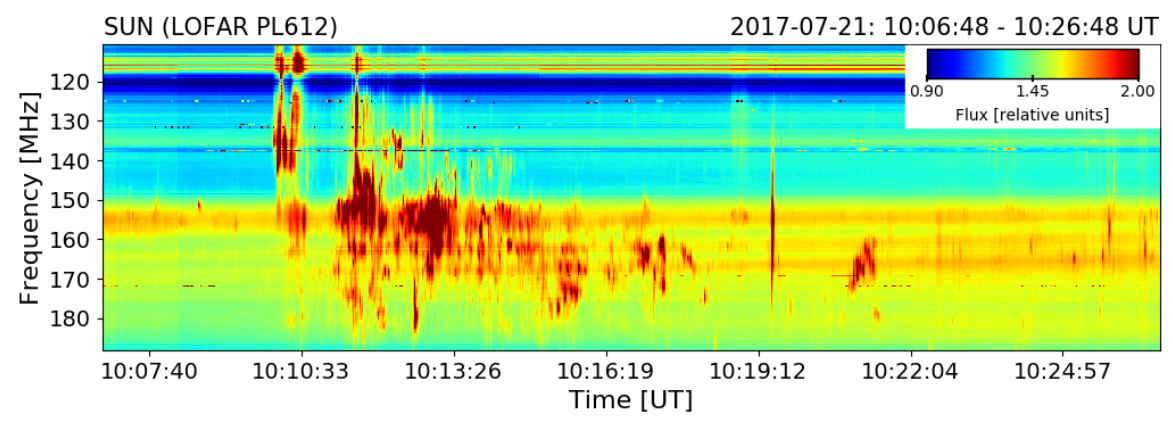

Figure 11: Solar dynamic spectra of the type I and III radio bursts recorded on 21 July 2017 between 10:06:48-10:26:48 UT in frequency bands 110.55-188.28 MHz.

Horst, A. van Houwelingen, A. Huijgen, M. Iacobelli, H. Intema, N. Jackson, V. Jelic, A. de Jong, E. Juette, D. Kant, A. Karastergiou, A. Koers, H. Kollen, V. I. Kondratiev, E. Kooistra, Y. Koopman, A. Koster, M. Kuniyoshi, M. Kramer, G. Kuper, P. Lambropoulos, C. Law, J. van Leeuwen, J. Lemaitre, M. Loose, P. Maat, G. Macario, S. Markoff, J. Masters, R. A. McFadden, D. McKay-Bukowski, H. Meijering, H. Meulman, M. Mevius, E. Middelberg, R. Millenaar, J. C. A. Miller-Jones, R. N. Mohan, J. D. Mol, J. Morawietz, R. Morganti, D. D. Mulcahy, E. Mulder, H. Munk, L. Nieuwenhuis, R. van Nieuwpoort, J. E. Noordam, M. Norden, A. Noutsos, A. R. Offringa, H. Olofsson, A. Omar, E. Orrú, R. Overeem, H. Paas, M. Pandey-Pommier, V. N. Pandey, R. Pizzo, A. Polatidis, D. Rafferty, S. Rawlings, W. Reich, J.-P. de Reijer, J. Reitsma, G. A. Renting, P. Riemers, E. Rol, J. W. Romein, J. Roosjen, M. Ruiter, A. Scaife, K. van der Schaaf, B. Scheers, P. Schellart, A. Schoenmakers, G. Schoonderbeek, M. Serylak, A. Shulevski, J. Sluman, O. Smirnov, C. Sobey, H. Spreeuw, M. Steinmetz, C. G. M. Sterks, H.-J. Stiepel, K. Stuurwold, M. Tagger, Y. Tang, C. Tasse, I. Thomas, S. Thoudam, M. C. Toribio, B. van der Tol, O. Usov, M. van Veelen, A.-J. van der Veen, S. ter Veen, J. P. W. Verbiest, R. Vermeulen, N. Vermaas, C. Vocks, C. Vogt, M. de Vos, E. van der Wal, R. van Weeren, H. Weggemans, P. Weltevrede, S. White, S. J. Wijnholds, 
T. Wilhelmsson, O. Wucknitz, S. Yatawatta, P. Zarka, A. Zensus, J. van Zwieten, LOFAR: The LOw-Frequency ARray, Astronomy \& Astrophysics 556 (2013) A2. arXiv:1305.3550, doi:10.1051/0004-6361/201220873.

[2] J. D. Turner, J.-M. Grießmeier, P. Zarka, I. Vasylieva, The search for radio emission from exoplanets using LOFAR low-frequency beam-formed observations: Data pipeline and preliminary results for the 55 Cnc system, ArXiv e-printsarXiv:1710.04997.

[3] A. Benz (Ed.), Plasma Astrophysics, second edition, Vol. 279 of Astrophysics and Space Science Library, Kluwer Academic Publishers, 2002. doi:10.1007/978-0-306-47719-5.

[4] Z. A. L. Sodré, R. D. Cunha-Silva, F. C. R. Fernandes, Analysis of Chains of Metric Solar Type I Bursts, Solar Physics 290 (2015) 159-168. doi: $10.1007 / \mathrm{s} 11207-014-0632-5$

[5] A. Warmuth, G. Mann, The Application of Radio Diagnostics to the Study of the Solar Drivers of Space Weather, in: K. Scherer, H. Fichtner, B. Heber, U. Mall (Eds.), Lecture Notes in Physics, Berlin Springer Verlag, Vol. 656 of Lecture Notes in Physics, Berlin Springer Verlag, 2005, p. 49.

[6] J. P. Wild, L. L. McCready, Observations of the Spectrum of High-Intensity Solar Radiation at Metre Wavelengths. I. The Apparatus and Spectral Types of Solar Burst Observed, Australian Journal of Scientific Research A Physical Sciences 3 (1950) 387. doi:10.1071/PH500387.

[7] H. Alvarez, F. T. Haddock, Solar Wind Density Model from km-Wave Type III Bursts, Solar Physics 29 (1973) 197-209. doi:10.1007/BF00153449

[8] M. J. Aschwanden, Physics of the Solar Corona. An Introduction, Praxis Publishing Ltd, 2004.

[9] L. Błaszkiewicz, W. Lewandowski, A. Krankowski, J. Kijak, O. Koralewska, B. Dąbrowski, Prospects for Scrutiny of Pulsars with Polish Part of LOFAR, Acta Geophys. 64 (1) (2016) 293-315. 
[10] B. P. Dąbrowski, A. Krankowski, L. Błaszkiewicz, H. Rothkaehl, Prospects for Solar and Space Weather Research with Polish Part of the LOFAR Telescope, Acta Geophys. 64 (3) (2016) 825-840.

[11] R. A. Fallows, W. A. Coles, D. McKay-Bukowski, J. Vierinen, I. I. Virtanen, M. Postila, T. Ulich, C.-F. Enell, A. Kero, T. Inatti, M. Lehtinen, M. Orispää, T. Raita, L. Roininen, E. Turunen, M. Brentjens, N. Ebbendorf, M. Gerbers, T. Grit, P. Gruppen, H. Meulman, M. J. Norden, J.-P. de Reijer, A. Schoenmakers, K. Stuurwold, Broadband meter-wavelength observations of ionospheric scintillation, Journal of Geophysical Research (Space Physics) 119 (2014) 10. arXiv:1511.00937, doi:10.1002/2014JA020406

[12] D. Mckay-Bukowski, Making Observations with Mode-357, Technical Note KAIRA-SGO-INF-011 (2013) 1-13.

[13] J. P. Wild, Observations of the Spectrum of High-Intensity Solar Radiation at Metre Wavelengths. II. Outbursts, Australian Journal of Scientific Research A Physical Sciences 3 (1950) 399-408. doi:10.1071/PH500399.

[14] J. P. Wild, Observations of the Spectrum of High-Intensity Solar Radiation at Metre Wavelengths. III. Isolated Bursts, Australian Journal of Scientific Research A Physical Sciences 3 (1950) 541-557. doi:10.1071/PH500541.

[15] J. P. Wild, K. V. Sheridan, G. H. Trent, The transverse motions of the sources of solar radio bursts, in: R. N. Bracewell (Ed.), URSI Symp. 1: Paris Symposium on Radio Astronomy, Vol. 9 of IAU Symposium, 1959, p. 176.

[16] A. Boischot, Caractères d'un type d'émission hertzienne associé à certaines éruptions chromosphériques, Academie des Sciences Paris Comptes Rendus 244 (1957) 1326-1329.

[17] G. Mann, F. Jansen, R. J. MacDowall, M. L. Kaiser, R. G. Stone, A helio- 
spheric density model and type III radio bursts, Astronomy \& Astrophysics 348 (1999) 614-620.

[18] F. Breitling, G. Mann, C. Vocks, M. Steinmetz, K. G. Strassmeier, The LOFAR Solar Imaging Pipeline and the LOFAR Solar Data Center, Astronomy and Computing 13 (2015) 99-107. arXiv:1603.05990, doi: $10.1016 / \mathrm{j}$. ascom.2015.08.001.

[19] C. Vocks, G. Mann, F. Breitling, Self-calibration strategy for a LOFAR solar radio burst observation, Astronomische Nachrichten 337 (2016) 1099. doi:10.1002/asna.201612443.

[20] D. E. Morosan, P. T. Gallagher, P. Zucca, R. Fallows, E. P. Carley, G. Mann, M. M. Bisi, A. Kerdraon, A. A. Konovalenko, A. L. MacKinnon, H. O. Rucker, B. Thidé, J. Magdalenić, C. Vocks, H. Reid, J. Anderson, A. Asgekar, I. M. Avruch, M. J. Bentum, G. Bernardi, P. Best, A. Bonafede, J. Bregman, F. Breitling, J. Broderick, M. Brüggen, H. R. Butcher, B. Ciardi, J. E. Conway, F. de Gasperin, E. de Geus, A. Deller, S. Duscha, J. Eislöffel, D. Engels, H. Falcke, C. Ferrari, W. Frieswijk, M. A. Garrett, J. Grießmeier, A. W. Gunst, T. E. Hassall, J. W. T. Hessels, M. Hoeft, J. Hörandel, A. Horneffer, M. Iacobelli, E. Juette, A. Karastergiou, V. I. Kondratiev, M. Kramer, M. Kuniyoshi, G. Kuper, P. Maat, S. Markoff, J. P. McKean, D. D. Mulcahy, H. Munk, A. Nelles, M. J. Norden, E. Orru, H. Paas, M. Pandey-Pommier, V. N. Pandey, G. Pietka, R. Pizzo, A. G. Polatidis, W. Reich, H. Röttgering, A. M. M. Scaife, D. Schwarz, M. Serylak, O. Smirnov, B. W. Stappers, A. Stewart, M. Tagger, Y. Tang, C. Tasse, S. Thoudam, C. Toribio, R. Vermeulen, R. J. van Weeren, O. Wucknitz, S. Yatawatta, P. Zarka, LOFAR tied-array imaging of Type III solar radio bursts, Astronomy \& Astrophysics 568 (2014) A67. arXiv:1407.4385 doi:10.1051/0004-6361/201423936.

[21] C. Mercier, P. Subramanian, G. Chambe, P. Janardhan, The structure 
of solar radio noise storms, Astronomy \& Astrophysics 576 (2015) A136.

arXiv:1412.8189, doi:10.1051/0004-6361/201321064. 